\title{
Nanoscale charge transport measurements using a double-tip scanning tunneling microscope
}

Philipp Jaschinsky, Jakob Wensorra, Mihail Ion Lepsa, Josef Mysliveček, and Bert Voigtländer

Citation: Journal of Applied Physics 104, 094307 (2008);

View online: https://doi.org/10.1063/1.3006891

View Table of Contents: http://aip.scitation.org/toc/jap/104/9

Published by the American Institute of Physics

\section{Articles you may be interested in}

Scanning tunneling potentiometry implemented into a multi-tip setup by software

Review of Scientific Instruments 86, 123701 (2015); 10.1063/1.4936079

Resistance and dopant profiling along freestanding GaAs nanowires

Applied Physics Letters 103, 143104 (2013); 10.1063/1.4823547

Nanomanipulation and nanofabrication with multi-probe scanning tunneling microscope: From individual atoms to nanowires

Review of Scientific Instruments 83, 063704 (2012); 10.1063/1.4727878

Local potentiometry using a multiprobe scanning tunneling microscope

Review of Scientific Instruments 79, 083704 (2008); 10.1063/1.2968111

Design and performance of a beetle-type double-tip scanning tunneling microscope

Review of Scientific Instruments 77, 093701 (2006); 10.1063/1.2336112

Variable-temperature independently driven four-tip scanning tunneling microscope

Review of Scientific Instruments 78, 053705 (2007); 10.1063/1.2735593

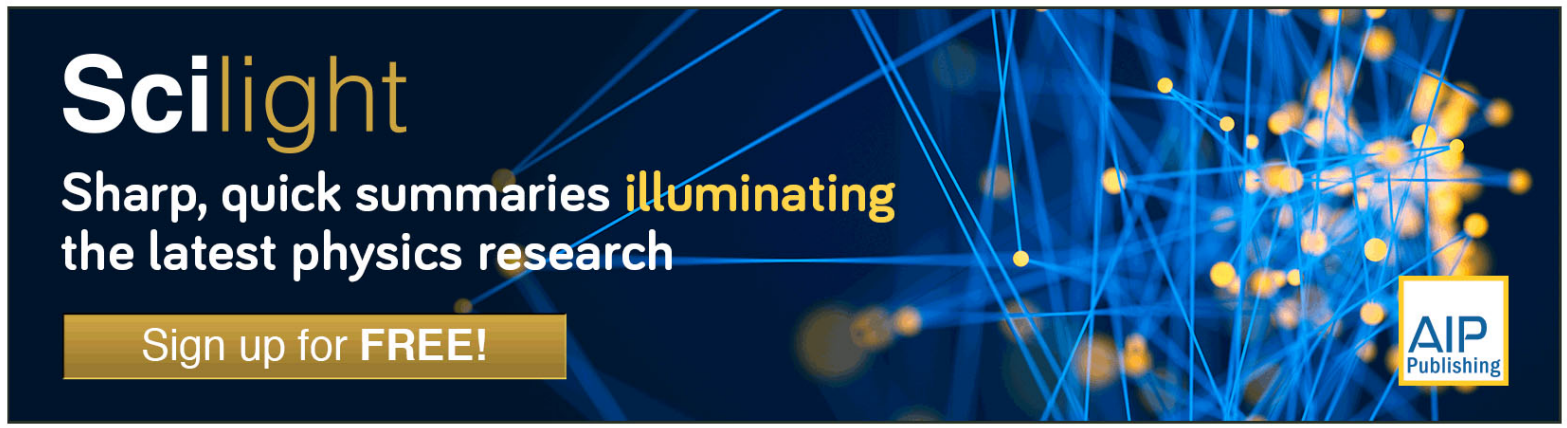




\title{
Nanoscale charge transport measurements using a double-tip scanning tunneling microscope
}

\author{
Philipp Jaschinsky, ${ }^{1,2}$ Jakob Wensorra, ${ }^{1}$ Mihail Ion Lepsa, ${ }^{1}$ Josef Mysliveček, ${ }^{1,3}$ and \\ Bert Voigtländer, ${ }^{1, a)}$ \\ ${ }^{1}$ Institute of Bio- und Nanosystems (IBN) and JARA-Fundamentals of Future Information Technology, \\ Forschungszentrum Jülich, D-52425 Jülich, Germany \\ ${ }^{2}$ Fraunhofer-Center of Nanoelectronic Technologies (CNT), Königsbrücker Str. 180, D-01099 Dresden, \\ Germany \\ ${ }^{3}$ Faculty of Mathematics and Physics, Department of Surface and Plasma Physics, Charles University \\ in Prague, CZ-18000 Prague, Czech Republic
}

(Received 13 May 2008; accepted 14 September 2008; published online 6 November 2008)

\begin{abstract}
We demonstrate the ability of a double-tip scanning tunneling microscope (STM) combined with a scanning electron microscope (SEM) to perform charge transport measurements on the nanoscale. The STM tips serve as electric probes that can be precisely positioned relative to the surface nanostructures using the SEM control and the height reference provided by the tunneling contact. The tips work in contact, noncontact, and tunneling modes. We present vertical transport measurements on nanosized GaAs/AlAs resonant tunneling diodes and lateral transport measurements on the conductive surface of $7 \times 7$ reconstructed $\mathrm{Si}(111)$. The high stability of the double-tip STM allows nondestructive electrical contacts to surfaces via the tunneling gaps. We performed two-point electrical measurements via tunneling contacts on the $\operatorname{Si}(111)(7 \times 7)$ surface and evaluated them using a model for the charge transport on this surface. (C) 2008 American Institute of Physics. [DOI: 10.1063/1.3006891]
\end{abstract}

\section{INTRODUCTION}

In the course of the ongoing miniaturization of electronic devices it is essential to investigate the charge transport through nanostructures. Besides the fabrication of useful nanostructures, a major challenge is the realization of electrical contacts. In the past, there have been a huge number of scientific studies of the charge transport measurements through nanostructures utilizing electrical contacts produced by lithographic means. ${ }^{1-4}$ However, nanostructures grown by self-assembly cannot be contacted lithographically in general because, first, their exact location on the surface is not known and, second, during the patterning of the electrodes the self-assembled nanostructures may suffer damage due to the chemical treatment of the samples.

Since its invention, the STM has been used as a powerful device to image and characterize nanostructures by spectroscopic means down to the atomic scale. ${ }^{5-8}$ However, one single probe is not sufficient to study charge transport along the surface. At least one additional probe is required to provide the second electrical contact. A multiprobe STM featuring several independent probes would not only be able to perform charge transport measurements but also to "drag and drop" objects at the nanometer scale. ${ }^{9}$

There have been several approaches integrating a multitip scanning tunneling microscope (STM) with up to four probes in ultrahigh vacuum (UHV) environment ${ }^{10-17}$ and charge transport measurements along the surface have been presented as well. ${ }^{18-23}$ In this publication we present charge transport measurements performed by a different kind of

${ }^{a)}$ Electronic mail: b.voigtlaender@fz-juelich.de. multitip STM-the coaxial beetle-type double-tip STM. ${ }^{24}$ This instrument has already proven its ability to image surfaces with atomic resolution due to its compactness. It is also able to manipulate the scanning tips independently of each other in a controlled manner by scanning electron microscope (SEM) navigation. Here, we will present charge transport measurements in the vertical and lateral directions performed by this instrument.

For the vertical transport measurements, nanometersized resonant tunneling diodes (RTD) were used. These are vertical GaAs/AlAs nanocolumns processed by the "topdown" approach. In the measurements, one STM tip provides a controlled Ohmic contact to the nanocolumn. The test current passes from this tip to the substrate. The second tip of the STM has been used as an isolated gate electrode generating an electric field to change the $I-V$ characteristic of the RTDs.

The lateral transport measurements were performed on a "self-patterned" $\mathrm{Si}(111)(7 \times 7)$ reconstructed surface. During the lateral transport measurement, the test current passes along the surface between the two STM tips. Both tips are kept in a tunneling contact with respect to the substrate. This prevents damage to both the tip and the surface reconstruction during the measurement. Moreover, the tunneling contacts have known (exponential) characteristics that allow us to describe the charge transport through the tunneling tips and along the surface with a two-point probe model. The distance-dependent tip-to-tip tunneling current along the $\mathrm{Si}(111)(7 \times 7)$ surface was compared to this model and transport parameters were derived. 

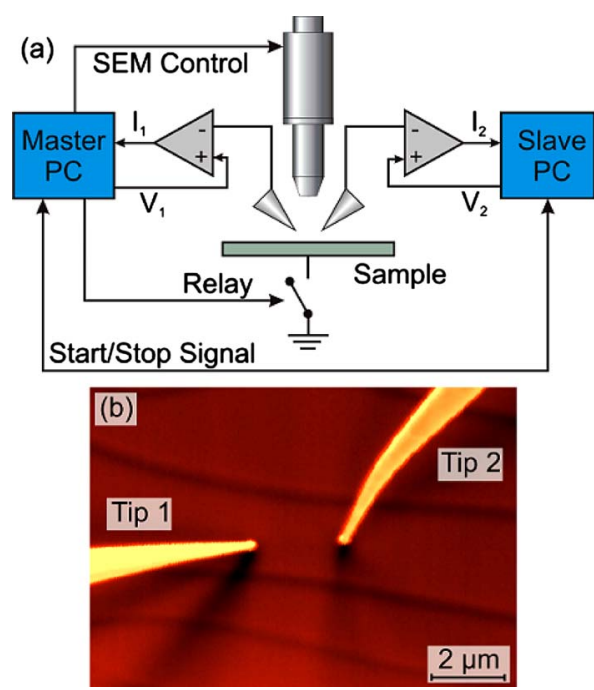

FIG. 1. (Color online) (a) Schematic drawing of the instrument configuration. The SEM column is in the center and images the two tips of the double-tip STM and the sample surface. The currents through the STM tips $I_{1}$ and $I_{2}$ are measured with two preamplifiers biased with $V_{1}$ and $V_{2}$, respectively. The master PC controls the STM tip 1, the SEM, and the sampleto-ground connection. The slave PC controls the STM tip 2. To assure a synchronous measurement of STM 1 and STM 2, PCs communicate start and stop signals. (b) SEM image of both STM tips in tunneling contact with a $\mathrm{Si}(111)$ surface. The visible structures on the surface are bunches of atomic steps formed during heating of the Si sample.

\section{EXPERIMENTAL}

The measurements were performed in a double-tip STM with two independently driven beetle-type STM units integrated in a small space. The STM exhibits a high stability enabling atomically resolved imaging with each tip. The open design makes the sample area easily accessible for SEM imaging. Details about the apparatus can be found in Ref. 24. A schematic drawing of the system is shown in Fig. 1(a). The SEM column in the center and both STM units are integrated within a UHV chamber with a base pressure of $\sim 1 \times 10^{-10}$ mbar. To exclude any additional current from the SEM during the charge transport measurements, the SEM beam can be blanked. Each STM is controlled by a dedicated personal computer (PC). The master PC controls the STM tip 1 [Fig. 1(a)], the SEM functions and the switching of the sample relay to disconnect the sample from the ground potential. The slave PC controls the second STM. The two PCs communicate via a digital input/output-line to enable synchronized measurements with both STMs. Each STM tip is connected to a biased preamplifier in order to apply independent potentials to the tips and, at the same time, to measure the current through the tips in a range from $10^{-4}$ to $10^{-10} \mathrm{~A}$. The STM tips point to the sample surface at an angle of $45^{\circ}$. The tips have been prepared by electrochemical etching of tungsten wires in $\mathrm{NaOH}$ solution and cleaned in situ by annealing under vacuum conditions.

\section{VERTICAL TRANSPORT MEASUREMENTS}

In this section, measurements on RTDs with lateral dimensions in the nanometer range are presented. The measurements were carried out on freestanding nanocolumns by contacting them mechanically with one STM tip. It will be

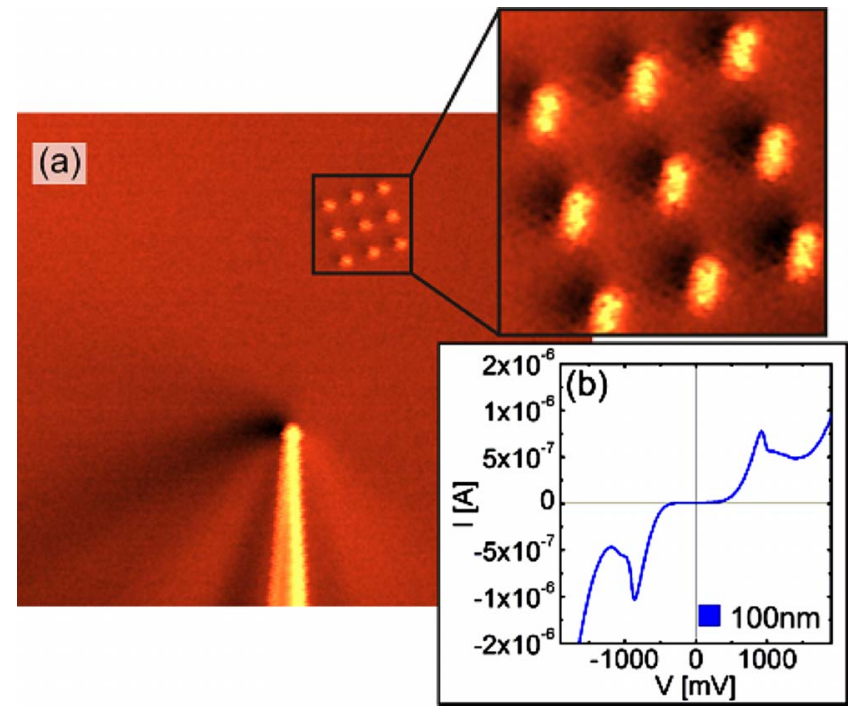

FIG. 2. (Color online) (a) SEM image of a STM tip in tunneling contact with the GaAs substrate close to an array of nine nanocolumns. The side length of a single nanocolumn is $100 \mathrm{~nm}$ and the height about $200 \mathrm{~nm}$. (b) $I / V$ characteristics of a $100 \mathrm{~nm}$ column measured by STM probing. Characteristic resonant tunneling peaks (at -865 and $+924 \mathrm{mV}$ ) are clearly visible. The PVRs are 2.2 on the left side and 1.6 on the right side, respectively.

shown that this method of studying nanosized devices by means of STM probing represents an alterative to lithographically processed contact pads.

The devices were fabricated by e-beam lithography and dry etching processes from a molecular beam epitaxy-grown layer stack. The heterostructure is based on GaAs with two embedded AlAs tunneling barriers (thickness: $1.7 \mathrm{~nm}$ ) separated by a GaAs quantum well (thickness: $5 \mathrm{~nm}$ ). Details of the layer structure can be found in Ref. 25. On top of the devices, a layer combination of low-temperature-grown $\mathrm{GaAs}$, titanium, and $30 \mathrm{~nm}$ gold provides an Ohmic contact to the GaAs-based nanocolumn. ${ }^{26}$

In Fig. 2(a), a SEM image of the columns with a lateral width of $100 \mathrm{~nm}$ and a height of $200 \mathrm{~nm}$ is shown. The STM tip is placed in tunneling contact close to the column array. To contact a single RTD nanocolumn, the higher resolution of the STM was used to locate the exact position of the columns by scanning the STM tip over the array. For contacting, the tungsten tip of the STM has to penetrate the gold layer on top of the nanocolumn. This is done by switching off the tunneling feedback and moving the STM tip into the sample in a controlled manner. Previous STM contact measurements on single nanocontacts revealed that the contact resistance between the STM tip and the gold layer depends on the penetration depth. It was found that the contact resistance becomes negligible with respect to the intrinsic resistance of the nanocolumn if a penetration depth of 10-20 nm has been reached. ${ }^{26}$ For the presented measurements, the penetration depth of $30 \mathrm{~nm}$ was used, i.e., the complete thickness of the gold layer. After establishing the Ohmic contact, a voltage ramp between the STM tip and the backside contact of the sample was applied. The current through the nanocolumn was measured with the STM preamplifier [Fig. 1(a) ]. The $I / V$ characteristics of a single $100 \mathrm{~nm}$ nanocolumn are shown in Fig. 2(b). The two pronounced peaks on the 

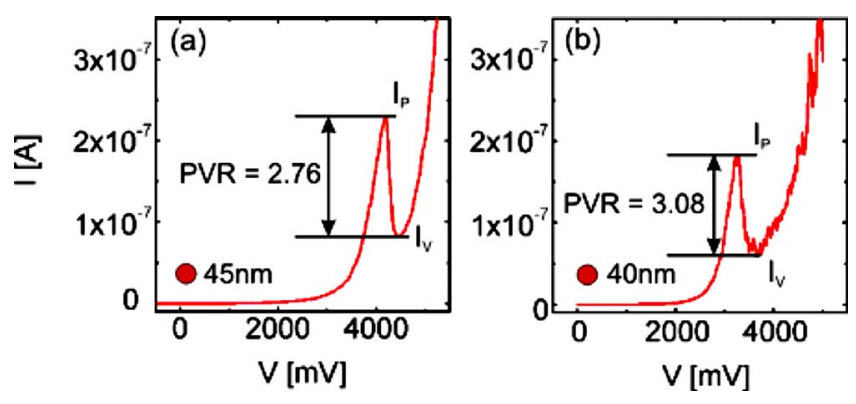

FIG. 3. (Color online) $I / V$ spectra for positive voltages of (a) a $45 \mathrm{~nm}$ RTD and (b) a $40 \mathrm{~nm}$ RTD. The PVR is a measure of the quality of the devices. The PVR in (a) was determined as 2.76 and in (b) 3.08.

positive and negative voltage side at +924 and $-865 \mathrm{mV}$ are typical for RTDs. This behavior results from a peaked transmission coefficient of the double-barrier structure in conjunction with the injected electron distribution from the emitter.

Using the above-described contacting method, we were able to characterize even smaller nanocolumns. Due to the high resolution of the STM, nanocolumns with a width down to $40 \mathrm{~nm}$ were measured. In Fig. 3, two $I / V$ characteristics of 45- and 40-nm-sized circular nanocolumns are shown. The higher intrinsic resistance of the smaller nanocolumns shifts the peaks to higher voltages. ${ }^{25}$ Only peaks at positive voltage are visible. Peaks at negative voltages are hidden in the exponential current increase in the diode itself. This asymmetry between positive and negative voltages can be explained by an increasing non-Ohmic behavior of the nanocontact on top of the RTD. ${ }^{26}$ The current peaks on the positive voltage side are highly pronounced. The device quality of a RTD is expressed by a peak-to-valley current ratio (PVR). The PVR for a $45 \mathrm{~nm}$ RTD is 2.76 and for a $40 \mathrm{~nm}$ RTD the PVR rises to 3.08 .

Our measurements show that it is possible to characterize nanosized electronic devices by probing with a STM tip. Since no further processing is needed to contact the devices, side effects influencing the physical properties and thus the $I / V$ curve of the devices do not apply.

In a next experiment, the second STM tip was used to study an electrostatic gate effect on the RTDs. The second STM tip was moved as close as possible to the first STM tip, which was already in mechanical contact with the nanocolumn. The schematic side view of the STM tips and the nanocolumn can be seen in Fig. 4(a) and the SEM top view in Fig. 4(b). The distance of the tips was estimated to be $D$ $\cong 200 \mathrm{~nm}$. The gate electrode (tip 2) has no electrical connection to the RTD device. To assure high electrical field strength in the vicinity of the double-barrier region, tip 2 was moved $30 \mathrm{~nm}$ in the $Z$-direction from its original position in the tunneling contact to the substrate.

The electrical measurements of a 70-nm-sized RTD using the gate electrode are shown in Fig. 4(c). Only the negative side of the $I / V$ characteristics is shown. The gate voltage was increased from -10 to $+10 \mathrm{~V}$ in four steps, and for each step a $I / V$ curve was measured. A small peak shift from 1480 $\mathrm{mV}$ (at $+10 \mathrm{~V}$ gate voltage) to $1680 \mathrm{mV}$ (at $-10 \mathrm{~V}$ gate voltage) can be observed. We explain the peak shift using the
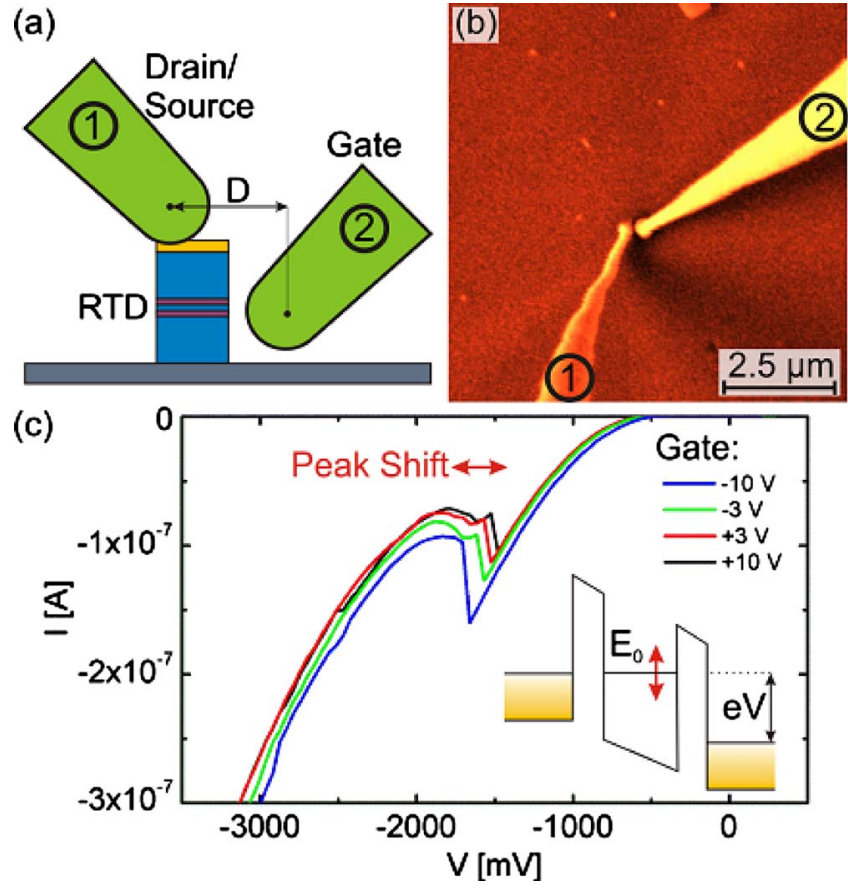

FIG. 4. (Color online) Gate effect measurements with the help of the second STM tip. (a) Schematic side view of the measurement setup. The RTD is contacted by tip 1 which can be seen as a source or drain electrode. The second STM tip (gate electrode) is placed at a distance of $D=200 \mathrm{~nm}$ from the double barrier of the RTD and lifted $30 \mathrm{~nm}$ from the substrate surface. (b) Top view of the measurement setup taken with SEM. (c) $I / V$ spectra of a 70-nm-sized RTD with different gate voltages. External electric field shifts the ground energy state of the electrons in the RTD as shown in the inset.

schematic band structure of the double-barrier region in the inset of Fig. 4(c). In the quantum well between the two AlAs barriers discrete electronic states are formed because of both the lateral and vertical electron confinements. We consider that in the electronic transport only the first level $E_{0}$ is involved. $E_{0}$ can be shifted relative to the emitter energetic spectrum by applying a sufficient gate voltage. A negative gate voltage will move the $E_{0}$ upwards, and vice versa.

The gate effect is quite small with a peak shift of 200 $\mathrm{mV}$ for a change in the gate bias of $20 \mathrm{~V}$ (approximately $1 \%)$. The following reasoning can explain the small size of the observed small peak shift. If we approximate the gate electrode as a conductive sphere, the potential at the place of the RTD can be approximated by the $1 / r$ dependence of the electrostatic potential of the gate electrode. This effect reduces the potential at the place of the RTD considerably compared to the potential of the gate electrode. Additionally the dielectric constant inside GaAs (13.5) induces a further reduction in the potential. This potential adds linearly to the potential induced by the source drain bias voltage inside the RTD and the peak shift is expected to be much smaller than the applied gate voltage.

\section{LATERAL TRANSPORT MEASUREMENTS}

In this section, we measure the charge transport along $\operatorname{Si}(111)-7 \times 7$ surface. Transport properties of the $\mathrm{Si}(111)(7$ $\times 7)$ surface are interesting to study since there is still a debate about its electronic character. It is not clear whether this surface is metallic due to the uneven number of electrons 


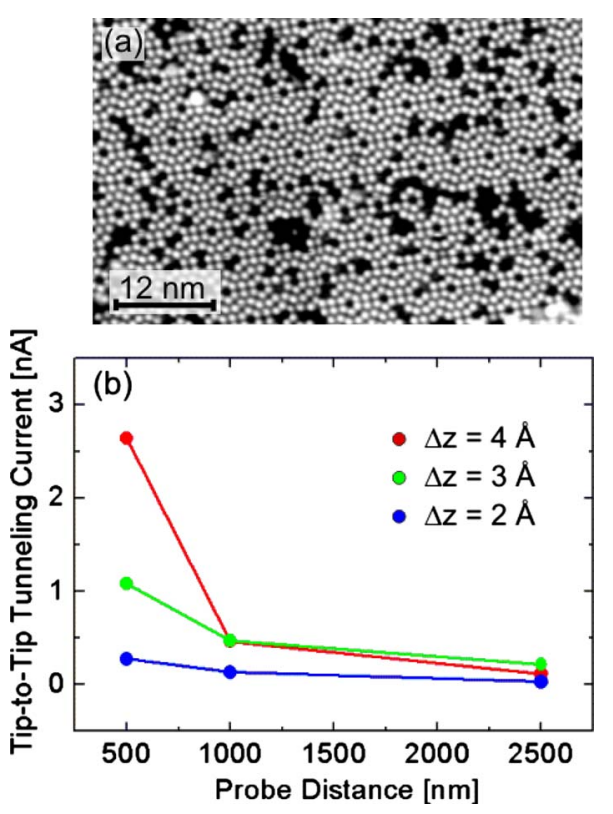

FIG. 5. (Color online) (a) STM image of atomically resolved $\mathrm{Si}(111)(7$ $\times 7)$ reconstructed surface. (b) Tip-to-tip tunneling current for three different tip heights: the tips were moved toward the surface by $\Delta z=2,3$, and $4 \AA$ for probe distances of $2.5 \mu \mathrm{m}-500 \mathrm{~nm}$.

per unit cell ${ }^{27,28}$ or insulating due to strong correlation effects. $^{29}$ There have been many attempts to estimate the surface conductance $\sigma_{s}$ of this surface ${ }^{30,31}$ including fourpoint probe measurements. ${ }^{32-35}$ Here we present a method for determining $\sigma_{s}$ of the $\operatorname{Si}(111)(7 \times 7)$ surface by using two STM tips that remain in tunneling contact. This method permits distance-dependent charge transport measurements without the destruction of the STM tips and the surface reconstruction. Moreover, due to the tunneling contacts the charge carriers are injected completely into the surface states of the $\mathrm{Si}(111)(7 \times 7)$ surface. ${ }^{31}$ This is not possible by mechanically contacting the surface because mechanical contacts penetrate the surface and destroy the surface reconstruction.

An $n$-type $\mathrm{Si}(111)$ wafer with a resistivity of $\rho$ $=5 \Omega \mathrm{cm}\left(n=9 \times 10^{14} \mathrm{~cm}^{-3}\right)$ was prepared using the standard high-temperature annealing to form the reconstructed surface. After checking the $\operatorname{Si}(111)(7 \times 7)$ reconstruction with one STM tip [Fig. 5(a)], the other STM tip was moved to a certain distance under SEM control. Before each measurement the same tunneling parameters were applied to both STMs $(I=0.5 \mathrm{nA}, V=-3 \mathrm{~V})$ and the SEM beam was blanked. The measurements were carried out for three tip-totip distances: $2.5,1$, and $0.5 \mu \mathrm{m}$. The measurement starts by turning off the feedback loops of both STMs and by opening the sample-to-ground switch [Fig. 1(a)]. To inject a current into the surface by one STM tip and collect it with the other STM tip, a voltage of $2 \mathrm{~V}$ was applied between the tips. To change the tunneling resistances between the tips and the surface, both tips were lowered in three steps $(\Delta z=2,3$, and $4 \AA$ A). During each step the tip-to-tip tunneling current was measured. The results are displayed in Fig. 5(b). The tip-totip tunneling current increases as the tips are lowered. This is due to the fact that the tunneling resistance between the STM

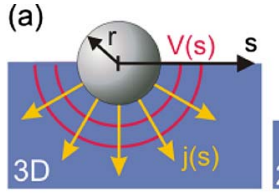

(b)

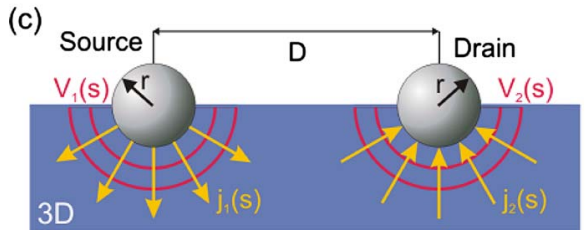

FIG. 6. (Color online) Voltage drop $V(\mathrm{~s})$ for a semi-infinite 3D conductor (a) and a 2D sheet (b) in a two-point probe arrangement. To derive the voltage drop between a current source and a current drain, a superposition of $V_{1}(\mathrm{~s})$ and $V_{2}(\mathrm{~s})$ has to be calculated. This is illustrated for the example of the $3 \mathrm{D}$ case $(\mathrm{c})$.

tips and the surface decreases as the vacuum gaps become smaller. The tip-to-tip tunneling current also increases as the probe distance becomes smaller.

To obtain quantitative information from the measurements in Fig. 5, we set up a model of the two-point probe measurement. First we treat the voltage drop along the surface. The actual charge transport at the Si surface generally proceeds via three channels: the two-dimensional (2D) surface conductivity layer of the $\operatorname{Si}(111)-(7 \times 7)$, a space charge layer (SCL), and the bulk [Fig. 7(b)]. We consider the two limiting cases, 2D and three-dimensional (3D) transport (Fig. $6)$. In the $3 \mathrm{D}$ case, the electrons enter the bulk of the sample (supposing a constant conductance $\sigma_{b}$ ) and the source and drain areas are considered as spherical electrodes, see Fig. 6(a). In the $2 \mathrm{D}$ case, the electrons remain at the sample surface and the source and drain areas are considered as cylindrical electrodes, see Fig. 6(b). Integrating the superimposed electric field of the source and drain areas [Fig. 6(c)], we obtain for the two-point surface resistance

$$
\begin{aligned}
& R_{3 \mathrm{D}}^{2 \mathrm{PP}}(\mathrm{D})=\frac{\Delta V_{3 \mathrm{D}}^{2 \mathrm{PP}}}{I}=\frac{1}{\pi \sigma_{b}}\left[\frac{1}{(r)}-\frac{1}{(\mathrm{D}-r)}\right], \\
& R_{2 \mathrm{D}}^{2 \mathrm{PP}}(\mathrm{D})=\frac{\Delta V_{2 \mathrm{D}}^{2 \mathrm{PP}}}{I}=\frac{1}{\pi \sigma_{s}} \ln \left[\frac{(\mathrm{D}-r)}{(r)}\right],
\end{aligned}
$$

for the 3D and 2D cases, respectively. The parameters determining the resistance are the bulk $\left(\sigma_{b}\right)$ or the surface $\left(\sigma_{s}\right)$ conductivities, the distance of the source and drain areas $D$, and the effective radius of the source and drain areas $r$. In the noncontact setup of our experiment the source and drain areas represent areas where the charge carriers injected/ extracted by tunneling move ballistically without significant energy losses. ${ }^{36}$ In the definitions of Eqs. (1) and (2) the resistance of these areas is implicitly zero.

During the two-point measurement, the surface resistance is in series with two tunneling resistances of the respective tunneling contacts, see Fig. 7(a). Thus, the current measured in the two-point measurement is

$$
I=\Delta U /\left(R_{T 1}+R^{2 \mathrm{PP}}+R_{T 2}\right),
$$

To make Eq. (3) useful for determining $\sigma_{b}$ or $\sigma_{s}$ we have to adopt certain assumptions regarding $R_{T}$. $R_{T}$ belongs to a tun- 


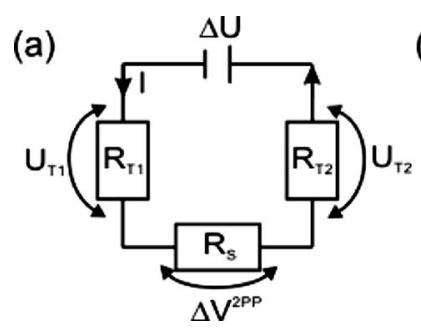

(b)

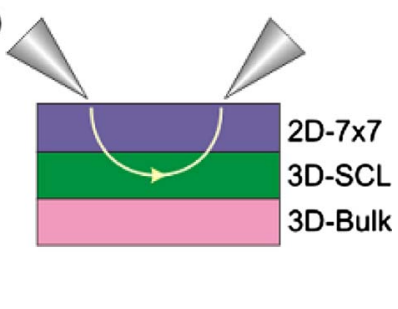

FIG. 7. (Color online) A simple scheme to calculate the tip-to-tip tunneling current. (a) Electrical circuit diagram of the two-point probe measurement. The applied voltage $\Delta U$ is composed of the tunneling voltages $U_{T 1}$ and $U_{T 2}$ and the voltage drop $\Delta V^{2 \mathrm{PP}}$ originating from the sample resistance $R_{S}$. (b) Three-layer model of the measured $\mathrm{Si}$ sample. The $\mathrm{Si}(111)(7 \times 7)$ surface is considered as a 2D conductor, whereas the space charge layer and the bulk are treated as a 3D conductor.

neling contact. Thus, in the approximation of small tunneling voltages it is an exponential function of $\Delta z \cdot{ }^{37}$ In the experiment, both STM tips are treated equally. Therefore, we consider

$$
R_{T 1}=R_{T 2}=R_{0} \exp (-2 \kappa \Delta z) .
$$

Equation (3), together with Eqs. (1) and (4), for the 3D case or alternatively Eq. (2) for the 2D case now yield the current $(I)$ as a function of two variables $D$ and $\Delta z$, and four parameters: $\sigma_{b}$ or $\sigma_{s}, r, R_{0}$, and $\kappa$. The parameters can be obtained by numerical least square fitting of the $I(D, \Delta z)$ dependence to the experimental data points in Fig. 5(b). We performed the fitting for both the $2 \mathrm{D}$ and $3 \mathrm{D}$ cases. The results of a fit for the 2D case are shown in Fig. 8. The 3D fit reproduces the experimental points comparably well. The fit quality does not allow deciding whether the $2 \mathrm{D}$ or the $3 \mathrm{D}$ transport mechanism dominates. However, a pure 3D transport can be excluded using other arguments given below.

The values of $\kappa, R_{0}(2 \AA)$, and $r$ obtained from a 2D fit equal the respective values from the $3 \mathrm{D}$ fit within $5 \% . \kappa$ $=0.9 \AA^{-1}$ is as expected for tunneling on semiconductors. ${ }^{38}$ $R_{0}$ has been determined for $\Delta z=2 \AA, R_{0}(2 \AA)=4 \mathrm{G} \Omega$. This is a plausible value in spite of the fact that the resistance of the tunneling junctions before the two-point measurement has been set to $6 \mathrm{G} \Omega$ and, given the above $\kappa$, a reduction by a factor of approximately 40 is expected upon lowering the tips by $2 \AA$. The tunneling tips are initially stabilized at a

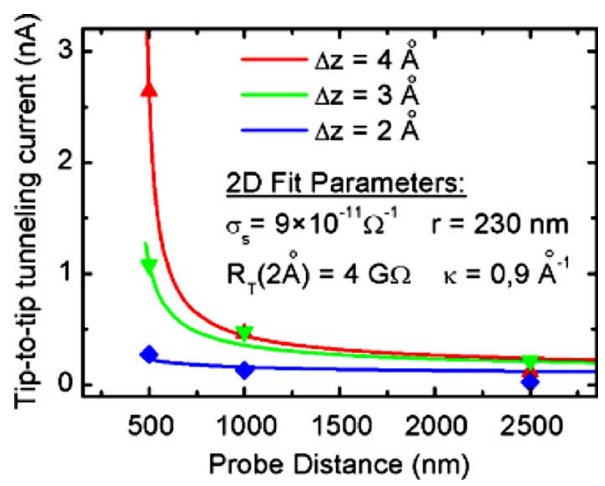

FIG. 8. (Color online) The tip-to-tip tunneling current calculated using the 2D two-point probe model in comparison to the measured data points. The free parameters $\sigma_{s} r, R_{0}$, and $\kappa$ were fitted to obtain the best match with the data points. tunneling voltage of $3 \mathrm{~V}$ far in a nonlinear part of the tunneling characteristic. During the two-point measurement the voltage across the tunneling junctions reduces to $<1 \mathrm{~V}$, approaching the linear regime. This is accompanied by an order-of-magnitude increase in the $R_{T}$ that compensates for the reduction in $R_{T}$ due to tip lowering. The effective radius of the source and drain areas has been fitted to $r=230 \mathrm{~nm}$. This value can be related to the decay length of the ballistic current in the source and drain areas. Measurements on semiconductors show that the decay length of the ballistic current is greater or equal than the corresponding inelastic mean free path. ${ }^{39}$ In our experiment, the bulk value of the electron mean free path (a majority carrier) at RT is approximately 50 $\mathrm{nm}$ for the used bulk doping level. ${ }^{40}$ The obtained $r$ $=230 \mathrm{~nm}$ suggests that deviations in the measured tip-to-tip current with respect to Eqs. (1) and (2) can be expected when $D$ becomes smaller than $500 \mathrm{~nm}$.

The surface conductivity resulting from the $2 \mathrm{D}$ fit is $\sigma_{s}$ $=9 \times 10^{-11} \Omega^{-1}$. This value is about two orders of magnitude lower than the conductivity determined in previous experiments. ${ }^{31,33}$ Compared to these experiments, in our surface conductivity measurement the test current is both injected and collected only via the surface states. This may eventually reduce the $3 \mathrm{D}$ contribution to the surface current measured in our two-point experiment and yield a lower $\sigma_{s}$ belonging mostly to the conductivity of the surface states. Another reason for this comparable low surface conductivity can be the high defect density of the $7 \times 7$ reconstructed surface obtained in our experimental setup [see Fig. 5(a)].

Alternatively, the bulk conductivity resulting from the $3 \mathrm{D}$ fit is $\sigma_{b}=1.8 \times 10^{-6} \Omega^{-1} \mathrm{~cm}^{-1}$. This value is far less than the nominal bulk conductivity of the used sample $\left(\sigma_{\text {sample }}\right.$ $\left.=0.2 \Omega^{-1} \mathrm{~cm}^{-1}\right)$. In the space charge region below the $\operatorname{Si}(111)-7 \times 7$ reconstructed surface, the conductivity decreases; ${ }^{33}$ however, the minimal value calculated for the given doping level using a Schottky approximation ${ }^{41}$ is 4 $\times 10^{-5} \Omega^{-1} \mathrm{~cm}^{-1}$, i.e., still more than an order of magnitude higher than the fitted one. This indicates that the charge transport through the $\mathrm{Si}(111)(7 \times 7)$ surface cannot be described by the 3D model in our approximation.

The presented lateral transport measurements represent a proof-of-principle study of the surface conductivity using a two-point measurement with two tunneling contacts. The issue of refining the $2 \mathrm{D}$ conductivity value might be resolved by a suitably designed follow-up experiment. The main improvement with respect to the presented measurements might be determining the $I-V$ characteristic of both tunneling junctions for each measurement of the tip-to-tip current. This would enable a direct access to the values of $R_{T 1}$ and $R_{T 2}$ and the possibility to determine $R_{s}$ without approximations used in this study.

\section{CONCLUSION}

In our work we have shown the ability of a combined SEM and a double-tip STM apparatus to perform charge transport measurements on the nanoscale under UHV conditions. Due to the coaxial beetle-type design of the double-tip STM it is possible to position the STM tips with a great 
freedom of movement by retaining the standard STM stability. These features were used to measure charge transport properties of single free standing nanosized RTDs. Furthermore, the charge transport properties along the $\operatorname{Si}(111)(7$ $\times 7$ ) reconstructed surface were studied by measuring the distance-dependent tip-to-tip tunneling current. A model for describing the two-point probe measurements for 3D and 2D conductors was introduced and used to derive the surface conductivity $\sigma_{s}$ of the $7 \times 7$ surface from the measured data.

\section{ACKNOWLEDGMENTS}

The authors would like to express their appreciation of the excellent technical assistance of Peter Coenen, Helmut Stollwerk, and the machine shop of IBN. J.M. acknowledges the support of the Ministry of Education of the Czech Republic, Project No. MSM 0021620834. We would like to thank Justin W. Wells for helpful discussions about the $\operatorname{Si}(111)(7 \times 7)$ surface.

${ }^{1}$ R. de Picciotto, H. L. Stormer, L. N. Pfeiffer, K. W. Baldwin, and K. W. West, Nature (London) 411, 51 (2001).

${ }^{2}$ Z. Zhong, D. Wang, Y. Cui, M. W. Bockrath, and C. M. Lieber, Science 302, 1377 (2003).

${ }^{3}$ B. Gao, Y. F. Chen, M. S. Fuhrer, D. C. Glattli, and A. Bachtold, Phys. Rev. Lett. 95, 196802 (2005).

${ }^{4}$ D. L. Klein, R. Roth, A. K. L. Lim, A. P. Alivisatos, and P. L. McEuen, Nature (London) 389, 699 (1997).

${ }^{5}$ B. C. Stipe, M. A. Rezaei, and W. Ho, Science 280, 1732 (1998).

${ }^{6}$ A. Yazdani, D. M. Eigler, and N. D. Lang, Science 272, 1921 (1996).

${ }^{7}$ V. J. Langlais, R. R. Schlittler, H. Tang, A. Gourdon, C. Joachim, and J. K. Gimzewski, Phys. Rev. Lett. 83, 2809 (1999).

${ }^{8}$ J. Mysliveček, A. Stróżecka, J. Steffl, P. Sobotík, I. Oštádal, and B. Voigtländer, Phys. Rev. B 73, 161302(R) (2006).

${ }^{9}$ P. Kim and C. M. Lieber, Science 286, 2148 (1999).

${ }^{10}$ M. Aono, C.-S. Jiang, T. Nakayama, T. Okuda, S. Qiao, M. Sakurai, C. Thirstrup, and Z.-H. Wu, Oyo Butsuri 67, 1361 (1998).

${ }^{11}$ H. Grube, B. C. Harrison, J. Jia, and J. J. Boland, Rev. Sci. Instrum. 72, 4388 (2001).

${ }^{12}$ I. Shiraki, F. Tanabe, R. Hobara, T. Nagao, and S. Hasegawa, Surf. Sci. 493, 633 (2001).

${ }^{13}$ X. Lin, X. B. He, J. L. Lu, L. Gao, Q. Huan, D. X. Shi, and H. J. Gao, Chin. Phys. 14, 1536 (2005).

${ }^{14}$ H. Okamoto and D. Chen, Rev. Sci. Instrum. 72, 4398 (2001).

${ }^{15}$ O. Guise, H. Marbach, J. T. Yates, Jr., M.-C. Jung, J. Levy, and J. Ahner,
Rev. Sci. Instrum. 76, 045107 (2005).

${ }^{16}$ K. Takami, M. Akai-Kasaya, A. Saito, M. Aono, and Y. Kuwahara, Jpn. J. Appl. Phys., Part 2 44, L120 (2005).

${ }^{17}$ T.-H. Kim, Z. Wang, J. F. Wendelken, H. H. Weitering, W. Li, and A.-P. Li, Rev. Sci. Instrum. 78, 123701 (2007)

${ }^{18}$ O. Kubo, Y. Shingaya, M. Nakaya, M. Aono, and T. Nakayama, Appl. Phys. Lett. 88, 254101 (2006).

${ }^{19}$ H. Okino, I. Matsuda, R. Hobara, Y. Hosomura, S. Hasegawa, and P. A. Bennett, Appl. Phys. Lett. 86, 233108 (2005).

${ }^{20}$ I. Matsuda, M. Ueno, T. Hirahara, R. Hobara, H. Morikawa, C. Liu, and S. Hasegawa, Phys. Rev. Lett. 93, 236801 (2004).

${ }^{21}$ X. Lin, X. B. He, T. Z. Yang, W. Guo, D. X. Shi, H.-J. Gao, D. D. D. Ma, S. T. Lee, F. Liu, and X. C. Xie, Appl. Phys. Lett. 89, 043103 (2006).

${ }^{22}$ S. Yoshimoto, Y. Murata, K. Kubo, K. Tomita, K. Motoyoshi, T. Kimura, H. Okino, R. Hobara, I. Matsuda, S. Honda, M. Katayama, and S. Hasegawa, Nano Lett. 7, 956 (2007).

${ }^{23}$ M. Ishikawa, M. Yoshimura, and K. Ueda, Jpn. J. Appl. Phys., Part 1 44, $1502(2005)$

${ }^{24}$ P. Jaschinsky, P. Coenen, G. Pirug, and B. Voigtländer, Rev. Sci. Instrum. 77, 093701 (2006).

${ }^{25}$ J. Wensorra, K. M. Indlekofer, M. I. Lepsa, A. Förster, and H. Lüth, Nano Lett. 5, 2470 (2005).

${ }^{26}$ J. Wensorra, M. I. Lepsa, K. M. Indlekofer, A. Förster, P. Jaschinsky, B. Voigtländer, G. Pirug, and H. Lüth, Phys. Status Solidi A 203, 3559 (2006).

${ }^{27}$ R. Losio, K. N. Altmann, and F. J. Himpsel, Phys. Rev. B 61, 10845 (2000).

${ }^{28}$ I. Barke, F. Zheng, A. R. Konicek, R. C. Hatch, and F. J. Himpsel, Phys. Rev. Lett. 96, 216801 (2006).

${ }^{29}$ D. Fick, C. Bromberger, H. J. Jaensch, O. Kuehlert, R. Schillinger, and C. Weindel, Surf. Sci. 600, 3835 (2006).

${ }^{30}$ Y. Hasegawa, I. W. Lyo, and P. Avouris, Surf. Sci. 357-358, 32 (1996).

${ }^{31}$ S. Heike, S. Watanabe, Y. Wada, and T. Hashizume, Phys. Rev. Lett. 81, 890 (1998).

${ }^{32}$ T. Tanikawa, K. Yoo, I. Matsuda, S. Hasegawa, and Y. Hasegawa, Phys. Rev. B 68, 113303 (2003).

${ }^{33}$ J. W. Wells, J. F. Kallehauge, T. M. Hansen, and Ph. Hofmann, Phys. Rev. Lett. 97, 206803 (2006).

${ }^{34}$ S. Hasegawa, I. Shiraki, F. Tanabe, R. Hobara, T. Kanagawa, T. Tanikawa, and I. Matsuda, Surf. Rev. Lett. 10, 963 (2003).

${ }^{35}$ F. Smits, Bell Syst. Tech. J. 37, 711 (1958).

${ }^{36}$ M. Prietsch, Phys. Rep. 253, 163 (1995).

${ }^{37} \mathrm{C}$. Julian Chen, Introduction to Scanning Tunneling Microscopy, 2nd ed. (Oxford University Press, New York, 2007).

${ }^{38}$ R. M. Feenstra, J. A. Stroscio, and A. P. Fein, Surf. Sci. 181, 295 (1987).

${ }^{39}$ R. Heer, J. Smoliner, G. Strasser, and E. Gornik, Appl. Phys. Lett. 73, 1218 (1998).

${ }^{40}$ L. Weber and E. Gmelin, Appl. Phys. A: Solids Surf. 53, 136 (1991).

${ }^{41}$ P. Jaschinsky, Ph.D. thesis, RWTH Aachen, 2007. 